\title{
Ciertos espacios de ocupación. La relación agroindustria-protestantismo en la formación de una región fronteriza entre México y Estados Unidos
}

\section{Some spaces of occupation. The agro-industry- Protestant relationship in the formation of a border region between Mexico and the United States}

Abbdel Camargo Martínez a* (D) https://orcid.org/0000-0002-8038-8089

a Universidad Nacional Autónoma de México, Instituto de Investigaciones Económicas, México, correo electrónico: abbdel@gmail.com

\section{Resumen}

En este documento se propone analizar la interrelación de dos dinámicas de ocupación territorial basada en el expansionismo estadounidense en una re-

Recibido el 15 de junio de 2018. Aceptado el 17 de octubre de 2018. Publicado el 12 de noviembre de 2018.

${ }^{*}$ Autor para correspondencia: Abbdel Camargo Martínez, correo electrónico: abbdel@gmail.com

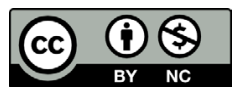

Esta obra está protegida bajo una Licencia Creative Commons Atribución-NoComercial 4.0 Internacional. gión agroindustrial del noroeste de México. Por medio de una perspectiva longitudinal este documento plantea cómo han actuado los procesos de colonización a través del desarrollo económico y la instauración de un modelo religioso de tipo protestante en el modelo productivo y en el poblamiento regional de una de las zonas agroexportadoras más importantes del noroeste del país. A pesar de limitaciones en el método, el trabajo sobresale al vincular dinámicas complejas de la globalización que usualmente son analizadas por separado. El trabajo muestra el desarrollo de un sentido colonial en la dinámica de ocupación territorial de empresas vinculadas a la agroindustria y al desarrollo de un campo religioso protestante en este territorio fronterizo; al mismo tiempo se identifica la disputa por la tenencia de una identidad regional que no ha estado exenta de conflictos entre los pobladores.

Palabras clave: ocupación, economía, protestantismo, poblamiento, región, frontera.

\begin{abstract}
This paper proposes to analyse the interrelation of two dynamics of territorial occupation based on American expansionism in an agroindustrial region of northwestern Mexico. The paper will analyze, from a longitudinal perspective, the colonization processes through economic development and the establishment of a Protestant re-
\end{abstract}

CÓMO CITAR: Camargo, A. (2018). Ciertos espacios de ocupación. La relación agroindustria-protestantismo en la formación de una región fronteriza entre México y Estados Unidos [Some spaces of occupation. The agro-industry-Protestant relationship in the formation of a border region between Mexico and the United States]. Estudios Fronterizos, 19, e017. doi:https://doi.org/10.21670/ ref. 1817017 
ligious model in the productive model and in the regional settlement of one of the most importantagro-exporting zones of the northwest of Mexico. Despite limitations in the method, the work excels in connecting complex dynamics of globalization that are usually analyzed separately. The work shows the development of a colonial sense in the dynamics of territorial occupation of companies linked to agro-industry and the development of a Protestant religious field in the borders; at the same time, it identifies the dispute over the possession of a regional identity that has not remained untouched by conflicts between the local settlers.

Keywords: occupation, economy, Protestantism, settlement, region, border.

\section{Introducción}

Históricamente, el desarrollo de las relaciones fronterizas entre México y Estados Unidos ha estado ligado a la dependencia económica y a una integración regional desigual. En la región agroexportadora del Valle de San Quintín, ubicada en el estado fronterizo de Baja California, se puede observar este proceso tanto en la dinámica productiva como en la historia del poblamiento regional, los cuales se han caracterizado por el impulso de una política expansionista proveniente de los Estados Unidos que se ha alimentado del desarrollo de un imaginario colonialista que ha elaborado la noción de un territorio baldío, factible de ser ocupado.

La expansión territorial es, desde el siglo xIx, una de las características más destacadas del desarrollo histórico de los Estados Unidos. Esta práctica —colonialestadounidense se establece como afirmación de una hegemonía ideológica en amplios territorios que fueron incorporados a esa nación por su cercanía geográfica, y que paulatinamente avanzó hacia una dominación colonial (Spurr, 2014). ${ }^{1}$ Para ello se desarrollaron procesos de asimilación y domesticación de los territorios y sus habitantes a través de mecanismos disciplinarios que involucran al territorio como espacio físico, a la tierra como entidad comercial y a sus moradores como piezas fundamentales para la organización de un modelo de producción particular. Los estudiosos tienden a coincidir en que esta práctica expansionista ha tenido una lógica comercial donde los estadounidenses salieron a buscar nuevos mercados dónde vender sus productos y a extraer fuentes de materias primas, lo que provocó la adquisición de colonias y la expansión extracontinental. Pero también ha tenido un sentido ideológico que enarbola un sentido de misión civilizadora, donde esa nación tenía que cumplir un papel entre razas consideradas como inferiores, lo que significa que la expansión del cristianismo era, ante todo, un mandato divino.

En el espacio fronterizo del noroeste de México, como espacio enfocado en la producción de alimentos frescos para los mercados mundiales, este imaginario se ha materializado en un proceso de integración regional desigual, que involucra a los dos países y que es evidente en el ámbito económico, sin embargo, postulamos que a la

\footnotetext{
${ }_{1}^{1}$ Piense el lector en la ampliación que se realizó hacia el sureste luego de la fundación de las trece colonias inglesas, por ejemplo: la anexión y disputa por Oregón (1819), la pérdida de más de la mitad del territorio de México (1848), y la compra de Alaska (1867). Así, contrario a lo que había sido la tradición estadounidense de incorporar territorios vecinos, los nuevos territorios fueron convirtiéndose en colonias de los Estados Unidos al incorporar, por ejemplo, a Hawai (1893) y Puerto Rico (1898).
} 
par de la expansión del capital en esta región fronteriza, se dio también la expansión de una ideología protestante en lo que ha sido un territorio históricamente misionero. Nuestra hipótesis es que ambos componentes — dinámica productiva y campo religioso protestante- determinan el desarrollo de un complejo transfronterizo donde se establecen jerarquías centradas en la producción de formaciones globales en campos concretos que son visibles a nivel local.

Al partir de la idea de que las afiliaciones religiosas producen perfiles religiosos regionales (Marroquín, 2007), y estos están determinados por procesos históricos de indole local, interesa establecer aquí la relación entre el ámbito religioso protestante y sus valores asociados con las estructuras económicas dominantes en la dinámica de producción agroexportadora, como muestra de la estructuración geopolítica de los componentes de la globalización contemporáneos que pueden establecerse en un territorio particular. Al mismo tiempo, al mostrar el proceso por etapas del poblamiento regional a través de la dinámica de estructuración productiva y la presencia de sociedades misioneras protestantes, permite establecer las formas de integración regional transfronteriza, su efecto en la estructura productiva y, en el desarrollo de una identidad regional en disputa.

¿Cómo han operado el ámbito productivo y religioso protestante en la definición de esta región transfronteriza? ¿Cómo en esta integración regional se fue desarrollado un territorio misional donde actúan múltiples sociedades religiosas internacionales? ¿Cómo estos dos campos han participado en el desarrollo de una de las regiones con mayor dinamismo económico y de diversidad étnica en el país?

Este trabajo establece una orientación longitudinal en su análisis y deriva de un trabajo de investigación más amplio (Camargo, 2015) donde se analizó la intersección entre el desarrollo de este enclave agroexportador y la internacionalización de un modelo religioso en el proceso de articulación comunitaria de un grupo indígena que se instaló en esta zona fronteriza. Los datos sociohistóricos que aquí se presentan se basan en la sistematización de entrevistas a pobladores del Valle de San Quintín, pastores y miembros de las iglesias realizadas en periodos intermitentes de trabajo de campo entre los años 2005 a 2010 y entre 2012 a 2014, así como en la revisión de fuentes documentales que describen los procesos de industrialización fronteriza, la revisión histórica de los modelos de misiones presentes en la península, los circuitos y patrones migratorios regionales y, en general sobre la dinámica del poblamiento fronterizo.

Este documento se divide en dos apartados. En el primero se describe el proceso de desarrollo de este enclave agroexportador, poniendo énfasis en la dinámica del poblamiento regional. En el segundo apartado se exalta la presencia misional en esta franja fronteriza definiendo dos modelos misionales que operan en la región y que se han articulado al perfil de sus pobladores. El documento cierra con una serie de reflexiones generales. 


\section{Desarrollo de un enclave agroexportador}

\section{Tierra de nadie que todos quieren}

La historia del poblamiento del Valle de San Quintín representa una muestra a nivel regional del proceso demográfico más amplio que se ha llevado a cabo en la península de Baja California, pues este proceso ha estado fuertemente ligado a la etapa de colonización basado en el sistema de misiones y al establecimiento de las relaciones fronterizas resultantes de la expansión capitalista en el suroeste norteamericano (Canales, 1995). Se puede adelantar que para el caso del Valle de San Quintín, estos dos elementos han determinado la forma del poblamiento regional.

Los documentos históricos señalan que los primeros intentos de colonización en la zona fueron hechos por religiosos dominicos, quienes al continuar la obra de evangelización de jesuitas y franciscanos, construyeron ocho misiones en la parte norte de la península de Baja California. En la región de San Quintín los dominicos fundan la misión Santo Domingo de la Frontera (ubicado en la actual delegación Vicente Guerrero), la cual funcionó de 1775 a 1839 (Velasco, Zlolnisky y Coubés, 2014). La presencia de los dominicos en San Quintín representa en los hechos el primer intento de colonización religiosa de la zona. ${ }^{2}$

A fines del siglo XVII, estas mismas misiones enfrentaron un proceso de crisis y desarticulación que adquirió impulso y manifestaciones concretas a partir de la independencia de México, cuando la economía regional comenzó aún con mayor fuerza el proceso de integración y subordinación al capitalismo norteamericano, sin que se diera una vinculación con la economía del centro del país (Canales, 1995, p. 8).

La idea de la península como una tierra de nadie, lejana, abandonada e inhóspita, ya se había concebido.

Si el poblamiento religioso se basó en un proceso de expansión misionera católica, el poblamiento civil se efectuó por un expansionismo económico que se sumó a uno territorial. A fines del siglo xIx, con el porfiriato, en la región se configuraron las condiciones de su poblamiento moderno, sin embargo, en este proceso ha habido una constante: la desatención institucional. Este abandono (político, económico y religioso) de la península marcó el derrotero histórico de una noción de territorio virgen propenso a ser colonizado, pues al aislamiento geográfico se sumó una debilidad demográfica que en conjunto contribuyeron a crear una imagen de la península de Baja California como una tierra deshabitada y salvaje, factible de ser ocupada. La noción de una "tierra de nadie" fue fraguada bajo un manto colonialista, la cual ha sido evidente en la forma histórica de su poblamiento y en la dinámica de colonización misional impulsada por múltiples sociedades religiosas a lo largo del tiempo.

\footnotetext{
${ }^{2}$ La orden de los dominicos alcanzó su mayor número de miembros durante la expansión del catolicismo en América, África y Asia en los siglos XVI al XVIII. Entre algunos de sus mayores representantes se ubican Fray Bartolomé de las Casas, Fray Antonio de Montesinos y Fray Pedro de Córdova.
} 


\section{San Quintín como una tierra de ocupación}

La primera ley de colonización para ocupar las tierras con familias extranjeras y mexicanas mediante la venta de tierra de Baja California a empresas extranjeras, fue adoptada por el presidente Benito Juárez en 1864 bajo el título de Ley de concesión sobre los terrenos baldíos de Baja California, la cual fue revocada en 1871. Nótese la exaltación de un terreno abandonado y poco aprovechado por parte del gobierno mexicano al denominar al territorio de la península como una tierra sin uso, a decir, baldía. Poco más tarde, durante el porfiriato, la nueva ley de colonización (1883) tuvo el respaldo del Estado para la llegada e instalación de colonos, sin embargo, la colonización de la península se pensó a partir de la presencia y establecimiento de compañías particulares (Heath, 2011; Piñera, citado en Velasco et al., 2014). Desde ese momento la política de poblamiento regional se basó en un sistema de concesiones que tenía como consigna el desarrollo económico del territorio y su poblamiento. Así, empresas como The International Company of Mexico, la cual logró tener a su disposición prácticamente toda la superficie que corresponde al actual estado de Baja California, pudo explotar sin reparo alguno, mares y tierras de la península. En realidad esta fiebre concesionista sirvió a los intereses particulares extranjeros en el marco del expansionismo norteamericano del siglo xix, poniendo al país en peligro de una mayor pérdida territorial con los intentos filibusteros por apropiarse del territorio (Taylor, 2000). ${ }^{3}$

Cuando el gobierno mexicano retiró la concesión, esta pasó a manos de la Compañía Inglesa quien se encargó de fortalecer la urbanización de Ensenada y el desarrollo de la Bahía de San Quintín. Con esta compañía se trazó el primer plano de la región, se construyeron algunas instalaciones clave, como las oficinas de la propia compañía, un hotel, un molino, se instaló el telégrafo y se concretizó el ferrocarril peninsular que cruzaría la península desde San Quintín hasta el Mar de Cortés y se conectaría con el de Sonora. Este proceso terminó en 1917, cuando la concesión de tierras fue cancelada por el presidente Venustiano Carranza.

Como se observa, el imaginario de las Californias para las empresas extranjeras, se basó en una mirada baldía del territorio peninsular, pues históricamente se le vio como un territorio virgen, carente de instituciones y de relaciones de producción tradicionales, así como con una población diezmada. Bajo esta visión, las relaciones derivadas de la vecindad geográfica con los Estados Unidos se desarrollaron y expandieron prácticamente sin más restricciones que aquellas que pudieran imponerle las condiciones naturales y geográficas peculiares de la región (Canales, 1995, p. 15). Un interés de expansión a nivel económico, territorial y espiritual llevó a gobiernos, empresas, pobladores y grupos religiosos provenientes del norte a tener una mirada franca puesta sobre esta zona. Desde entonces, quizá aquella frase que se escuchó en el Senado norteamericano durante el penúltimo periodo del presidente Roosevelt (quien gobernó de 1933 a 1945) puede sintetizar esa visión estratégica de importancia geopolítica de la zona: "Baja California es solamente un lujo para México; pero para nosotros (Estados Unidos) es una necesidad” (citado en Jordán, 1997, p. 126). La

\footnotetext{
${ }^{3}$ En el siglo XVIII se les llamaba filibusteros a los aventureros (piratas) que sin comisión de gobierno alguno invadían territorios ajenos a mano armada. En México William Walker fue el más reconocido de los denominados filibusteros del siglo XIX, al tratar de conquistar — sin éxito- los territorios de Sonora y Baja California.
} 
proximidad geográfica favorecía la dinámica expansionista y una vinculación natural con ese país y, por razones obvias, el mayor impacto lo recibió la región fronteriza del norte de México (Figura 1).

Figura 1. Ubicación del Valle de San Quintín

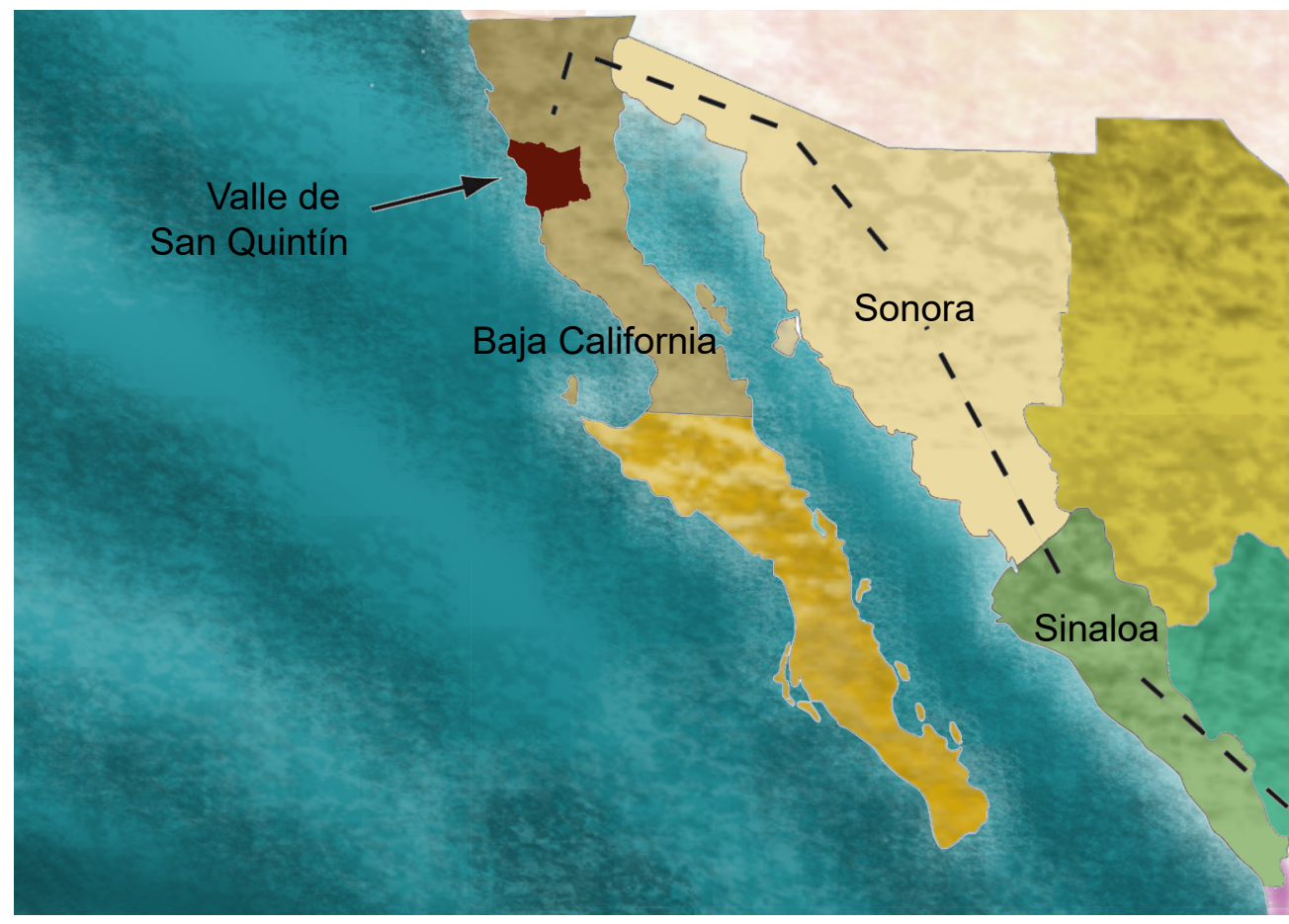

Fuente: Elaboración propia.

\section{Los primeros residentes. La noción del migrante emprendedor}

La política de concesiones de fines del siglo xıx trató de atraer a nuevos pobladores, por lo que la Compañía para el Desarrollo de Baja California ${ }^{4}$ se dio a la tarea de atraer a mil colonos que se involucrarían en la construcción de un molino de harina, de las vías del ferrocarril que partía de San Quintín a Ensenada y la concreción de un servicio postal que tenía como destino la ciudad de San Diego en California, sin embargo, para 1885 solo se habían logrado incorporar 200 personas (Moyano, citado en Moreno, 2008).

\footnotetext{
${ }^{4}$ De acuerdo con Taylor (2000, p. 54):
}

Durante el periodo de consolidación política en el Distrito Norte bajo la jefatura del coronel Esteban Cantú Jiménez y los gobiernos posrevolucionarios de la década de 1920, en la región se crearon ciertas bases para fomentar su desarrollo general, así como estrechar sus lazos con el resto de la República. Cantú, quien había sido nombrado como gobernador y comandante militar del distrito por la Convención revolucionaria en enero de 1915, durante el periodo que gobernó adoptó varias medidas para fomentar el desarrollo del Distrito y de sus pobladores, persuadiendo a Carranza para que declarara caducas las concesiones que el gobierno de Porfirio Díaz había otorgado a la Mexican Land and Colonization Company y a la Lower California Development Company (Compañía para el Desarrollo de Baja California). 
Ya a inicios del siglo xx con la reforma agraria, familias que habían sido repatriadas de los Estados Unidos o que se habían quedado en la zona fronteriza, así como hombres provenientes de diversos estados del interior del país como Michoacán y Jalisco, o de otras regiones que habían sido desplazados del reparto agrario cardenista, fueron los primeros pioneros que se asentaron en las "tierras de trabajo" de San Quintín. Desde entonces, un espíritu pionero de los primeros pobladores que llegaron desde otras latitudes a esta tierra de labor, fraguó una noción nativista del esfuerzo y el trabajo como cualidades esenciales del espíritu emprendedor de la zona, tal y como los valores protestantes exaltan. Por nativismo, y siguiendo a Higham (1983, p. 4 ) "entendemos a la oposición intensa a una minoría de origen extranjero cuya presencia, a juicio de quien discrimina, amenaza de alguna manera la identidad o seguridad de la mayoría”. Así, los primeros pobladores se montaron sobre una visión exacerbada del migrante emprendedor quien, como fruto de su esfuerzo, se otorga la cualidad de los pobladores pioneros quienes tienen derechos sobre la tierra y su usufructo.

Si el desarrollo de una política de colonización basado en concesiones pudiera describir toda la dinámica poblacional de la zona en esa época (siglo xıx), un segundo momento del poblamiento de San Quintín fue posible gracias a un impulso pionero desarrollado por los primeros pobladores asentados en la región (primera parte del siglo xx). ${ }^{5}$ Ya para mediados de la década de 1930:

El gobierno mexicano decidió dar destino a las tierras que habían recobrado de la concesión dada tanto a los americanos como a los ingleses. En esa época 500 familias fueron repatriadas de Estados Unidos y enviadas al Valle de la Esperanza, como también fue llamado el Valle de San Quintín. ${ }^{6}$

A pesar de estos esfuerzos, el aislamiento geográfico (pues aún no había carretera) todavía reinaba en la zona, por lo que la dinámica de poblamiento de la región seguía basándose en el esfuerzo individual. Bajo esta perspectiva, el abandono institucional, entre ellos el religioso, mantenía a la zona bajo el velo desértico de un imaginario del territorio desaprovechado.

Para el año de 1947 se realiza el trazado de la terracería hasta el kilómetro 300 desde Tijuana, lo cual reduce un poco el tiempo de transporte desde la ciudad, pero esta terracería pronto quedó dañada y nuevamente se retomaron los caminos antiguos (Ramírez, 2008, p. 53). Para 1950 se tiene ya registro de la presencia de los primeros migrantes que iniciaron una insípida agricultura comercial, aunque aún en la década de 1960 la región de San Quintín continuó siendo una zona muy poco poblada, con ranchos pioneros y menos de 4000 habitantes en toda la región, considerada desde Punta Colonet hasta San Quintín (Velasco et al., 2014). Hasta entonces se mantenía el perfil del poblador emprendedor quien, como cowboy moderno se mantenía expuesto al talante oportunista de su esfuerzo inquebrantable. Esta fue la idea de un sujeto sacrificado, proveniente de las tierras del norte de California -Estados Unidos-, quien cruzó la frontera con una noción de ocupar el territorio vacío. A este perfil de

\footnotetext{
${ }^{5}$ Aun cuando hay registros que para el proyecto de construcción del ferrocarril que iría de Tijuana y hasta Bahía de los Ángeles, y que tendría como centro nodal la bahía de San Quintín, hablan de que "poco más de 175 hombres fueron contratados, 90 de ellos mexicanos que venían del sur, (entre los cuales había indígenas yaquis), y los demás extranjeros, incluyendo chinos" (Heath, 2011, p. 211).

${ }^{6}$ La noción de "esperanza" acompañó precisamente la creación impetuosa del carácter emprendedor de la identificación nativista de esta porción del territorio peninsular.
} 
emprendedor se sumó aquel otro perfil proveniente del occidente de México, quien llegó a construir, poblar y "levantar" a toda una región. Así personas de Michoacán, Jalisco y Guanajuato comenzaron a ocupar las primeras tierras de forma permanente y a desarrollar una (aún) incipiente agricultura comercial.

Este perfil de actor regional inició con esos primeros rancheros inmigrantes quienes con el paso del tiempo devinieron en empresarios locales sobre quienes se comenzó a gestar una identidad regional definida por un arraigo territorial excluyente a las subsecuentes oleadas de migrantes. Este proceso se ha observado en los estudios de migración e identidad, donde los inmigrantes más antiguos se posicionan como los nativos y las olas sucesivas vienen a representar a los extranjeros. Aquí los que llegan atentan contra lo "establecido" y entra en juego una diferenciación de tipo racial, especialmente si los que arriban proceden de lugares considerados como menos prósperos, menos "desarrollados" o menos "civilizados" (Guarnizo, 2010, p. 48). Como mencionamos líneas arriba, este proceso recibe el nombre de nativización y lo entendemos como el arraigo poblacional paulatino, que implica apego a un territorio con un sentido de apropiación excluyente respecto a los recién llegados (Ngai, citado en Velasco 2011, p. 48). Piense el lector que los grupos que sucedieron a esta oleada de primeros migrantes - mestizos pioneros-, fueron precisamente los grupos indígenas provenientes de amplias regiones empobrecidas del sur del país. Es así que el componente racial se ha mantenido como elemento definitorio de la dinámica socioeconómica en la región.

Esta noción nativista del migrante emprendedor pervive hasta nuestros días y es parte del relato que define la identidad regional, donde productores locales o actores regionales enrolados en el comercio y los servicios se posicionan como los "auténticos" fundadores de una región que, con base en su esfuerzo y trabajo ha salido a flote. ${ }^{7}$ Un ejemplo de lo anterior lo encarna el Rancho Los Pinos, ${ }^{8}$ perteneciente a los Hermanos Rodríguez, quienes exaltan ese espíritu emprendedor signado en la figura del padre como "el gran iniciador". De acuerdo al relato familiar, a principios de la década de 1950 la familia Rodríguez Hernández arribó a la zona proveniente del estado de Michoacán. El padre, quien fue un inmigrante campesino pobre, logró con su esfuerzo y trabajo individual construir una de las empresas más exitosas de la zona. La materialización de esta noción nativista se aprecia en el cementerio familiar donde se ha edificado la estatua del padre — fundador — donde se exhibe la figura de un hombre con un notable perfil campesino, que usa un sombrero de palma roído y que con un azadón en mano se dispone a labrar la tierra. De igual forma, en uno de sus campamentos agrícolas más grandes en toda la zona, se exhibe un mural donde se observa a este mismo hombre de origen campesino, trabajando la tierra y sembrando tomates en los prósperos campos del valle que él había forjado. Desde entonces la identidad regional se ha ido fraguando bajo una diferenciación social tanto de clase como étnica, de la cual hoy día somos testigos.

\footnotetext{
${ }^{7}$ Esta noción de la identidad fragmentada del sanquintinense, permeó la lucha por la municipalización del Valle de San Quintín como sexto municipio del estado.

${ }^{8}$ Productora Industrial del Noroeste (Pinos) es una de las empresas más grandes de la zona que acapara buena parte de la producción regional.
} 


\section{Presencia indígena y la noción del migrante temporal}

Con la irrupción del siglo xx se generó en la región un particular estilo de desarrollo de las relaciones fronterizas entre México y los Estados Unidos, mismas que tuvieron impacto en la dinámica del poblamiento regional y en los patrones migratorios (Zlolniski, 2011). Un imaginario particular se ha consolidado a partir del perfil de actores que han definido la noción del desarrollo regional. Como se ha mencionado, a partir de la década de 1950 algunas familias iniciaron una incipiente agricultura enfocada hacia la exportación, pero no fue sino hasta las décadas de 1960 y 1970 que se da un verdadero auge en esa actividad. Particularmente a partir de la década de 1970, en el noroeste del país se consolidaron enormes empresas hortícolas, a menudo con vínculos de colaboración e inversión con empresas extranjeras, como resultado de los procesos de integración comercial y por la cercanía geográfica con los Estados Unidos. Así:

Debido a su posición estratégica, los estados del noroeste han manteniendo relaciones comerciales con los Estados Unidos, razón por la cual esta zona recibió fuertes inversiones privadas y públicas, nacionales y extranjeras, enmarcadas en el proceso de restructuración productiva que se había iniciado ya en el país (Velasco et al., 2014, p. 31).

Esta orientación productiva modificó el perfil del tipo de migrante que arribaría a la zona. Del lado mexicano, a partir de la década de 1970, el intrépido colono pionero que llegó a labrar la tierra y con ello un futuro promisorio, dio paso al migrante indígena, a ese trabajador temporal y avecindado, ${ }^{9}$ el cual llega huyendo de las severas condiciones de pobreza que priva en sus estados de origen como Guerrero y Oaxaca (Zabin, 1992). El estatus emprendedor del sujeto nativista de los primeros rancheros de las décadas de 1930 y hasta 1960 culminó con la presencia del trabajador dependiente encarnado por la figura del jornalero agrícola e indígena que irrumpió a partir de la década de 1970 y que siguió arribando a la región durante dos décadas más (1980 y 1990). ${ }^{10}$ Desde entonces la oposición de la identidad regional entre empresarios-rancheros y jornaleros-migrantes ha definido un campo de disputa entre los actores regionales.

Del lado norteamericano, por su parte, el agrobusiness abrió camino al empresario capitalista, a ese ranchero estadounidense, también extranjero, que saca provecho de la debilidad institucional regional y que demandaba crecientes contingentes de mano de obra para una industria de alimentos en expansión. En este contexto la composición étnica de la mano de obra en mercados laborales altamente competitivos, se ha definido por agregar a la oferta de su fuerza de trabajo, su disponibilidad para incorporarse productivamente como fuerza de trabajo móvil, lo que es adaptable a las exigencias variables del mercado y es donde descansa buena parte de su capacidad competitiva (González, 2009).

\footnotetext{
${ }^{9}$ Los avecindados son aquellos individuos o familias que también llegan a vivir a las comunidades establecidas, y se integran a su vida comunal, pero en diferente posición y estatus. Una suerte de extranjería permea permanentemente su condición.

${ }^{10}$ Esta población indígena, de origen migrante, fue principalmente mixteca, zapoteca y triqui de Oaxaca, pero también hay presencia de mixtecos y nahuas de Guerrero, nahuas de Veracruz, purépechas de Michoacán, huicholes de Nayarit y de los grupos mayas del estado de Chiapas, entre otros, lo que convierte a San Quintín como una de las regiones con mayor plurietnicidad del país.
} 
En un análisis retrospectivo se puede afirmar siguiendo a Velasco et al. (2014, p. 71), que la incorporación del Valle de San Quintín al mercado internacional se puede comprender por etapas: La fase inicial (que comprende la década de 1970 e inicios de la década de 1980) se define por el desarrollo de una infraestructura que comunicó a la región con la frontera, pues con la finalización de la carretera transpeninsular en 1973, los primeros empresarios pronto iniciaron la producción de tomate para el mercado norteamericano. La segunda fase es la de expansión (que va de mediados de la década de 1980 hasta mediados de la década de 1990) y está definida por la expansión de la agricultura a gran escala, la integración de la economía regional a la estadounidense y por la instalación de grandes compañías que generaron un proceso de producción y modernización tecnológica. La última fase, que sería de restructuración productiva (que va de mediados de la década de 1990 y hasta nuestros días), se caracteriza porque las empresas transitan de la producción de alto volumen a la producción de calidad con base en la innovación tecnológica (introducción de invernaderos y sistemas por goteo), la diversificación de cultivos y la ampliación del ciclo de cosechas. ${ }^{11}$ Estos cambios explican la adaptación histórica de una región a un mercado de alimentos frescos a nivel internacional.

A la creciente intervención de capital extranjero en la zona, se sumó una progresiva participación de grupos de iglesias, también extranjeras, que vieron en la región una oportunidad de posicionar un proselitismo religioso de tipo protestante basado en el trabajo misionero, que abonaba al relato del esfuerzo individual que define la primera etapa del poblamiento regional. De hecho, podemos señalar que la dinámica del poblamiento de la región de San Quintín también incluyó momentos de un expansionismo religioso, anclado este a grupos de iglesias norteamericanas organizadas bajo un esquema misional (Camargo, 2011). Ello significa que el desarrollo y consolidación de la región de San Quintín tiene un viso histórico definido tanto por la integración económica regional como por la dinámica misional que le ha otorgado su cualidad fronteriza.

\section{La presencia misional protestante en San Quintín}

Usualmente, el protestantismo estadounidense ha sido un vehículo progresivo de la ideología político-religiosa del Destino Manifiesto. Como se sabe, el Destino Manifiesto es una doctrina que expresa la creencia de que Estados Unidos está destinado a expandirse por destino divino. Así, se cree que la expansión no solo es justa, sino también obvia (pues es manifiesta) y certera (pues cumple un destino).

[Se] propaga entonces la convicción de que la misión que dios dio al pueblo estadounidense fue explorar y conquistar nuevas tierras, con el fin de llevar

\footnotetext{
${ }^{11}$ A partir del año 2002 la producción total basada en el sistema de invernaderos empieza a crecer hasta llegar a cerca de 100000 toneladas, lo que corresponde a 35\% de la producción total en 2008. Su implantación es particularmente notoria durante el ciclo primavera-verano, cuando la producción en invernadero representa $44 \%$ del total (Gallardo, 2010).
} 
a todos los rincones la luz de la democracia, la libertad y la civilización. ${ }^{12}$ Esta ideología ha justificado el intervencionismo en la política de otros países, así como la expansión territorial mediante la guerra, la economía o la religión (Marín, 1982, p. 125).

De igual manera que la economía o la religión, interesa aquí explicar el expansionismo protestante en las tierras bajas de California.

\section{El despunte de la agricultura en la región y el primer modelo misional}

Luego del debacle de las Misiones de los Dominicos católicos en la región de San Quintín a fines del siglo xvII, diversos grupos misioneros irrumpieron de manera esporádica en la zona. Esta primera etapa de la actividad misional se desarrolló entre los siglos xviI y XviII, a través de la implantación de un modelo proselitista de tipo colonial, donde se observan las Misiones históricas del catolicismo colonial y la presencia de grupos protestantes que participaron del poblamiento regional en la expansión del proselitismo cristiano. Este primer modelo misional se extendió hasta fines del siglo xix e inicios del siglo xx cuando se observó de manera permanente la presencia de otros grupos religiosos en la región con un perfil protestante, derivado de la vecindad geográfica con los Estados Unidos. Los registros señalan que el de los mormones fue uno de estos primeros asentamientos religiosos que se establecieron en la zona. En el año de 1909, familias pertenecientes a la Iglesia First Born of the Fullnees of Times se instalaron en el Valle de San Quintín, lugar donde fundaron la colonia agrícola Zarahemla, ubicada en la Delegación Vicente Guerrero. Según la Iglesia de Jesucristo de los Santos de los Últimos Días (mormones) estas personas fueron expulsadas de sus filas por defender la práctica de la poligamia (Hernández, 1999). ${ }^{13}$

La práctica misional de esa época se basó en la implantación de una orientación religiosa de tipo protestante entre los pobladores mestizos (provenientes del occidente de México) recién llegados a la región. Ya señalamos que:

El poblamiento de las principales localidades que integran el Valle de San Quintín fue realizado a finales de 1950, gracias al reparto de tierras efectuado por el gobierno federal. Las primeras familias mexicanas de inmigrantes que llegaron al Valle procedían de Guanajuato, Michoacán y Jalisco. ${ }^{14}$ Poblar el

${ }^{12}$ Marín (1982, p. 120) nos deja ver un breve episodio de dicho Manifiesto donde se señala:

El cumplimiento de nuestro destino manifiesto es extendernos por todo el continente que nos ha sido guardado por la Providencia para el desarrollo del gran experimento de la libertad y el autogobierno; es un derecho como el que tiene el árbol de obtener aire o la tierra para el desarrollo pleno de nuestras capacidades de crecimiento que tenemos como destino.

${ }^{13}$ En la actualidad a este ejido se le conoce en toda la región por el asentamiento que ahí tienen los mormones, lo que habla de la capacidad de sobrevivencia de este nicho religioso en la región.

${ }^{14}$ De acuerdo con datos de Canales (1995) entre 1930 y 1960, uno de los periodos de mayor crecimiento de la entidad, más de $50 \%$ de la población residente del estado era originaria de otros estados de la República. 
Valle de San Quintín representó un gran reto para los primeros inmigrantes; no había escuelas ni ningún tipo de servicio de atención médica, y bienes como víveres, ropa y enseres domésticos se conseguían a precios exorbitantes. Para acceder a esa zona se requería atravesar un tramo de la Sierra de San Pedro Mártir y utilizar un estrecho camino de unos 200 kilómetros de terracería. El aislamiento en que se encontraba San Quintín hizo que esa región fuera escasamente atendida por la Iglesia católica (Hernández y O’ Connor, 2013, p. 13).

De igual manera, fue desatendida por las instituciones de gobierno (Hernández, $2000)^{15}$ Los sacerdotes católicos que visitaban el Valle procedían de Ensenada y sus recorridos por la zona eran esporádicos. Así, dado que desde la época colonial la Iglesia católica no fue capaz de consolidar su presencia institucional en la zona, debido principalmente a lo escasa de la población y lo exiguo de su desarrollo económico-, no resulta sorprendente que esta región fuera un terreno más abierto y accesible para los protestantes estadounidenses y sus iglesias (Jaimes, 2009). La noción de una "tierra nueva al sur de la frontera" motivó a individuos, grupos independientes, y organizaciones religiosas norteamericanas a hacer visitas periódicas y a establecer la noción de un territorio misional en las tierras llanas de Baja California.

Así, contrariamente al abandono de la Iglesia católica, desde mediados de la década de 1950 varias sociedades misioneras protestantes provenientes de los Estados Unidos emprendieron una incansable tarea para "atender" a la población que se encontraba en la región. Los primeros en establecerse fueron los bautistas. Su principal labor fue la construcción de una clínica-hospital para la atención a personas de escasos recursos. A estos les siguieron los misioneros de "Cristo por su Mundo", organización evangélica que establecería un ambicioso proyecto de asistencia para niños sin hogar y que ha tenido un importante impacto en atención a huérfanos, madres solteras, adultos mayores, personas con discapacidad y problemas de adicciones (Zlolniski, 2011). También aparecerían células de grupos misioneros protestantes independientes provenientes de otras zonas de la entidad, pero pertenecientes a asociaciones religiosas en los Estados Unidos, quienes comenzaron a hacer trabajo proselitista sobre una población aún diezmada. De este modo, el relato del emprendedor nativista se vio fortalecido con el arribo de una fe cristiana de tipo protestante a la zona, misma que engendraría otro relato, el de la "prosperidad" como un rasgo sobresaliente de la dinámica fronteriza empresarial.

La aparición de lo que se puede llamar como economía religiosa, se encuentra articulada a una producción orientada al mercado, donde el discurso ya no se construye tanto para producir una verdad sino para asegurarse un éxito. Este proceso implica la instauración de una narrativa basada en el éxito, tal y como se aprecia entre los discursos de los primeros habitantes que paulatinamente fueron escalando a empresarios locales.

De esta forma, el reto del primer modelo misional en la zona consistió en construir un proyecto de evangelización de largo plazo que permitiera la conversión de los

\footnotetext{
${ }^{15}$ La visión de Baja California como un territorio despoblado, aislado y susceptible de colonizar, permeó todo el siglo XIX. Solo se modificó hasta que la expansión capitalista estadounidense, el crecimiento urbano de San Diego, después de la Guerra del 47 y del tratado de Guadalupe Hidalgo impulsó el nacimiento de núcleos poblacionales en el norte de la península (Moyano, citado en Jaimes, 2009).
} 
nuevos colonos, en su mayoría fervientes católicos procedentes del centro-occidente de México, y con una vigorosa tradición popular. Don José Hernández fue una de las primeras almas conquistadas por los misioneros bautistas; originario de Yuriria, Guanajuato, este ejidatario dejó atrás su adicción al alcohol para abrazar la fe evangélica y más tarde, se convertiría en uno de los primeros pastores mexicanos establecidos en esta parte sur del municipio de Ensenada (Hernández, 1999).

\section{La expansión de la agroindustria en la región y el segundo modelo misional}

Es importante señalar que a diferencia de los estudios de cambio religioso en sociedades indígenas donde se habla de cómo estas sociedades religiosas se trasladan a los territorios tradicionales con el fin de implantar la nueva fe, el proceso que aquí exponemos no tiene un sentido unidireccional que describa el mecanismo donde unos grupos llevan la fe cristiana a lugares donde antes no la había. Desde San Quintín registramos un proceso diferenciado, y por demás relevante; aquel donde la presencia de sociedades religiosas internacionales logró instaurar un entorno protestante en una región fronteriza al que diversos grupos indígenas se tuvieron que adherir y sobre el cual han tenido que realizar una serie de adecuaciones a sus sistemas normativos.

A partir de la década de 1970, con un mercado de trabajo agrícola en plena expansión, la labor proselitista de estas sociedades religiosas tuvo que ser reorientada y adaptada al perfil de la población que masivamente había irrumpido en la zona: los trabajadores indígenas migrantes. Estamos así, frente a una de las formaciones religiosas protestantes que Lalive (1970) ya había distinguido en función de las grandes migraciones trasatlánticas: Las iglesias-diáspora que fundamentalmente combinan las tres dimensiones fundamentales del inmigrante: nacionalidad, religión y etnia. De acuerdo con el autor, debido al éxodo las poblaciones se han separado de su base y se constituyen como minorías étnicas en los nuevos espacios de arribo, formulándose de esta manera un sentido nativista que facilita la práctica colonial.

Postulamos que la segunda etapa del modelo misional en la región se basó en la implantación de un enfoque étnico en el proselitismo religioso derivado de la presencia de población indígena migrante en la zona. Desde su arribo a los campamentos en el Valle de San Quintín, los grupos indígenas que llegaban a la región a laborar en las tareas del campo, recibieron la atención de los misioneros evangélicos, por lo que la principal estrategia de acercamiento fue la dispensa de recursos asistenciales en la práctica misional.

Grupos de voluntarios pertenecientes a organizaciones evangélicas, entre los que se podían encontrar médicos, dentistas, enfermeras, profesores, estudiantes y amas de casa, establecieron año con año campamentos para profesar la palabra de dios de manera temporal en San Quintín. Caravanas de vehículos, procedentes de los Estados Unidos y Canadá, recorrían los campamentos donde permanecían los jornaleros para ofrecerles comida y ropa usada, proporcionarles atención médica, proyectarles películas de contenido cristiano y entregarles un mensaje de salvación (Hernández y O’Connor, 2013, p. 13). 
Para muchos de los jornaleros ese sería su primer contacto con la doctrina evangélica.

Si el primer modelo misional se basó en el uso de la palabra para acercarse a los primeros pobladores, el segundo modelo misional fue fincado sobre un tipo de proselitismo marcadamente asistencialista y el uso de recursos tecnológicos como medio exitoso de la propagación de esta nueva fe. En esta labor, estos grupos encontraron varios obstáculos al tratar de convertir a esta masa de trabajadores migrantes según Hernández y O’Connor (2013, p. 13) "Algunos misioneros que trabajaron en la región han señalado lo difícil que fue para ellos 'conquistar almas entre los indígenas'”. Los primeros grupos de trabajadores en arribar a la zona fueron los mixtecos de Oaxaca. Los relatos señalan que entre los principales obstáculos que limitaban su trabajo misionero se encontraban el que miembros de este grupo:

se acercaban a recibir donativos, participaban en las funciones de cine y recibían consultas médicas, pero eran muy reservados al tratar el tema de sus prácticas religiosas. La otra barrera importante era la del idioma. Las casas misioneras no contaban con pastores que conocieran alguna de las lenguas indígenas y solo un reducido número de jornaleros sabían hablar y leer en español (Hernández y O’́Connor, 2013, p. 13).

Por lo que estos grupos debieron desarrollar diversas estrategias para "alcanzar" a esta población.

A partir de entonces un persistente y metódico trabajo misionero que incluyó el estudio y comprensión de las lenguas indígenas, la formación de pastores indígenas respetando sus propias lenguas, la traducción de los textos y pasajes sagrados, así como diversas ayudas y apoyos materiales a las familias comenzaron a tener impacto entre la población indígena jornalera; todo este esfuerzo contribuyó a la contextualización de las nuevas iglesias en el suelo misionero de San Quintín. Esta práctica no debe pensarse que fue desarrollada únicamente por aquellas familias o grupos organizados de misioneros que provienen de iglesias locales en Estados Unidos o Canadá; hablamos más que nada de sociedades religiosas internacionales que funcionan como empresas religiosas globales y que actúan en los territorios. ${ }^{16}$

La presencia permanente de estos grupos de iglesias se dio a la par de la ampliación de los tiempos de trabajo de la población en este mercado laboral, que derivó en el asentamiento de estos trabajadores y la formación de colonias marginales en toda la región (Camargo, 2012). ${ }^{17}$ Como se ha mencionado, la presencia permanente de

\footnotetext{
${ }^{16}$ Un ejemplo de este tipo de sociedades misioneras internacionales presentes en San Quintín es el grupo Buenas Nuevas, que es filial de la organización Global Recordings Network (GRN) la cual ha hecho grabaciones y traducciones de la vida de Cristo en más de 6000 lenguas de todo el mundo desde el año de 1939 en que se fundó. Esto significa que en todo este tiempo han hecho traducciones de al menos una lengua por semana. Esta cifra es de considerase si tomamos en cuenta que, en todo el mundo hay poco más de 12000 lenguas, por lo que esta organización ha cubierto poco más de la mitad de las lenguas y dialectos que se hablan en todo el planeta.

17 Desde la década de 1980, en San Quintín han ocurrido profundas transformaciones económicas y demográficas, derivadas de los cambios que ha habido en la industria hortícola y por el establecimiento de miles de jornaleros agrícolas y sus familias en colonias de trabajadores. Este proceso de asentamiento de la población es relativamente reciente y ya podemos dar cuenta de sus implicaciones en la transformación demográfica de esa porción del territorio mexicano. Tan solo entre 1970 y el 2000 la población del Valle creció de manera vertiginosa con tasas medias de $7.2 \%$ anual, transitando de 8559 habitantes en 1970 a 92177 en el año 2010 (Instituto Nacional de Estadística y Geografía [Inegi], 2010; Velasco et al., 2014).
} 
trabajadores agrícolas devino del desarrollo económico e integración de la región de San Quintín con el capital extranjero, donde el modelo misional también se tuvo que adaptar. La estrategia de evangelización fincó en la noción de expansionismo territorial su estrategia proselitista, esto es, en la adaptación de la práctica protestante al perfil residencial de su población objetivo: las colonias formadas por los trabajadores. Un proselitismo itinerante en las principales delegaciones de la región y en los campamentos y colonias donde residía la población trabajadora, definió un protestantismo regional que basó su estrategia de evangelización inicial en la presencia continua de los misioneros en los campos de trabajo, para dar paso a la canalización de recursos económicos y la asistencia social en las colonias posteriormente formadas. Solo una vez resueltos los problemas locales más apremiantes (lotificación, construcción de viviendas, escuelas y clínicas) las iglesias tuvieron mayor eco en estos nuevos territorios indígenas, pues con el nacimiento de colonias en toda la región, también las iglesias lograron expandir su presencia. La búsqueda de nuevas posibilidades de trabajo y vivienda generaron al mismo tiempo la renovación de los espacios protestantes en los núcleos residenciales anclados a la formación de las colonias, donde la eclosión de iglesias locales consolidaron un liderazgo indígena que ha sido capaz de aglutinar un universo simbólico donde participan los signos asociados al protestantismo con aquellos otorgados por el catolicismo de la costumbre pero dando lugar también a la práctica pentecostal. En un sitio donde la presencia de las instituciones estatales ha sido débil, el papel de estas iglesias ha resultado fundamental. ${ }^{18}$ En este contexto, la Iglesia católica ha podido hacer muy poco para contrarrestar la influencia protestante en la zona (Hernández, 1999).

Habrá que insistir en que este arribo masivo de población en la región ha formulado una noción de extranjería a la presencia de esta población indígena trabajadora que no comparte la incipiente identidad regional que se basa en una noción nativista del migrante pionero, mestizo y emprendedor encarnado en los primeros pobladores, rancheros y empresarios de la zona. El relato dominante señala que los migrantes indígenas son producto del esfuerzo de esos primeros hombres, y en la lógica de estos pioneros, esta masa trabajadora resulta ser un apéndice poblacional necesario para el desarrollo económico del lugar, pero es carente del espíritu emprendedor que demanda la fragua de la identidad regional. Es este un discurso racial, más que de tipo moral.

Finalmente, habrá que resaltar que en el análisis del desarrollo de los mecanismos sociales que han acompañado el proceso de arraigo de esta masa trabajadora en la región - con las movilizaciones sociales, los intercambios comunitarios y la defensa de sus derechos-, se puede advertir que la apertura religiosa de estos grupos

\footnotetext{
${ }^{18}$ El espacio residencial definido por las colonias de trabajadores en San Quintín, modificó el perfil histórico del poblamiento regional, pues estas últimas migraciones tienen la característica de ser masivas, inducidas por una dinámica mercantil y ajenas a un esfuerzo gubernamental. El carácter masivo de crecimiento de la zona se vincula con el crecimiento estatal, pues el siglo XX se caracterizó porque la dinámica de poblamiento de todo el estado de Baja California se centró por el crecimiento demográfico explosivo basado en el arribo de importantes flujos migratorios que, atraídos por el desarrollo industrial fronterizo, se fueron empleando ya sea en la maquila o en el desarrollo de la agricultura comercial. Según datos de Canales (1995, p. 12) entre 1910 y 1990 la entidad creció a una tasa anual promedio de $6.4 \%$, cifra muy superior al promedio nacional y al del resto de los estados fronterizos $(2.1 \%$ y $2.6 \%$, respectivamente). Tal ritmo hizo que la población de Baja California se duplicara cada 11 años, mientras que la población del país lo hacía cada 33.
} 
ha representado una forma de acción política, y puede ser considerada como una experiencia novedosa de movilización indígena (Bengoa, 2000). Como señala Lalive (1970, p. 48) "el protestantismo y en particular el pentecostalismo se presenta como una respuesta religiosa comunitaria al abandono de grandes capas de la población en situación de exclusión y marginación social". Por tanto planteamos que, la participación de esta masa trabajadora en estas iglesias no responde a un sentido meramente doctrinario, sino a un modo consistente de concebir la posibilidad de mejorar las condiciones de vida, al plantearse la resolución de necesidades apremiantes. Este proceso de apropiación del universo protestante por parte de los grupos indígenas de la región no ha estado ajeno de conflictos entre familias y comunidades, sin embargo, es posible aventurar que en la definición de la religiosidad indígena contemporánea en esa región se vienen sumando las prácticas evangélicas y pentecostales como una forma de resistencia y continuidad de los elementos que constituyen lo étnico. Lo sincrético en este territorio fronterizo permite, entonces, la convergencia de partes antiguas de cosmovisiones transformadas por la conquista hispánica, reformuladas en el catolicismo popular y ahora rediseñadas con los elementos que el pentecostalismo proporciona a la construcción civil, religiosa y política de buena parte de los grupos indígenas asentados en el Valle, quienes al apropiarse de los valores pentecostales están "produciendo" comunidad y están logrando poner límites a la dinámica colonial y extractivista en que se sustenta el modelo agroexportador.

\section{Conclusiones}

Las migraciones, tanto históricas como las más recientes a la zona del Valle de San Quintín, en el estado de Baja California, han contribuido a la constitución de una región que basa las ventajas de su competitividad, en su ubicación estratégica y en el uso intensivo de mano de obra que se adecua a las necesidades del mercado (Lara, 2011). Más allá de la dinámica del perfil productivo, ha sido también el pluralismo religioso el que ha orientado la definición de una región donde se pueden observar la operación de campos globales en un territorio transfronterizo particular.

Históricamente, en San Quintín, el universo del protestantismo fue primeramente aceptado por la población asentada en los centros delegacionales existentes, configurada por población mestiza, residencialmente dispersa y con un relativo poder adquisitivo estable, derivado de una industria agrícola naciente, quienes desarrollaron un sentido de apropiación nativista respecto al territorio de labor de San Quintín. No fue hasta entrada la segunda mitad del siglo xx, con el repunte y posicionamiento de la zona como región hortícola de exportación, en que la presencia masiva de los trabajadores indígenas también trajo consigo un mayor activismo de estos grupos religiosos en los campos agrícolas y en las colonias nacientes. Esto es que la asimilación del protestantismo en la zona reconoce las características locales de poblamiento del territorio, así como del dinamismo de su economía.

La presencia en masa de trabajadores indígenas ha contribuido a la creación de campos de disputa en la definición de la identidad regional en términos de clase como étnicos. Esta disputa se ha intensificado a partir de la presencia permanente de mano de obra indígena en los espacios residenciales de la región, como resultado de los procesos de modernización tecnológica que posibilitó el empleo permanente 
de esta masa proletaria. La presencia indígena reorientó, además, la dinámica evangelizadora que habían desarrollado sociedades religiosas internacionales quienes habían implementado un metódico trabajo de evangelización como parte de la dinámica misional y de la labor expansionista estadounidense. A partir de la llegada en masa de la población jornalera en la década de los 70, la pluralización religiosa en la región abría paulatinamente su cauce a través de la presencia continua de los grupos de misioneros y su trabajo de asistencia social con la población ahí presente. Un proselitismo itinerante en los sitios de trabajo y residencial ha ido definiendo un protestantismo regional de tipo asistencial, que es capaz de adaptarse tanto a las condiciones histórico-locales como del perfil de su feligresía.

El papel de estas denominaciones en la región en general y en las colonias en particular, ha tenido una importancia fundamental en el proceso de cambio residencial pues con la creación de redes relacionales y organizacionales en las colonias, cuyos elementos son las cruzadas de evangelización, la presencia de las organizaciones transnacionales con una base local, las conferencias de pastores, los medios de comunicación y el núcleo cristiano de ayuda a desamparados, aseguran la presencia continua de estas sociedades religiosas, su expansión y la aceptación de la nueva fe en esta región transfronteriza. La instalación de este tipo de protestantismo en las colonias de trabajadores indígenas, corona un modelo de evangelización donde empresas religiosas globales han operado a través de un modelo misional focalizado.

Desde esta perspectiva, la presencia de población indígena en el noroeste de México, particularmente en regiones con las características del Valle San Quintín, no solo ha permitido la consolidación de su participación económica en los mercados internacionales (Martínez, 2006), sino que también ha dispuesto la posibilidad de consolidar un protestantismo de tipo étnico en esa región (Camargo, 2011), el cual ha ido moldeando una religiosidad local que se nutre de la identidad étnica, y que se inscribe en los procesos de recomposición del campo religioso de las comunidades indígenas del país.

Consideramos que el contexto religioso protestante al cual se incorporaron estos contingentes de indígenas, ha tenido importantes impactos en el proceso de gestión de la identidad religiosa de estos grupos, así como de sus formas organizativas en ese nuevo sitio de asentamiento. Dado que la coexistencia de una diversidad cultural y étnica puede ser reforzada por la presencia de una heterogeneidad religiosa (Garma, 2004), consideramos que, en San Quintín, debemos hablar del carácter contextual de la definición de estas comunidades de fe y de la vitalidad que estas adquieren en los territorios fronterizos (Demera, 2008). Es así que nuestro enfoque se articula con aquellos estudios donde el protestantismo y las múltiples ofertas religiosas, favorecen el despliegue de estrategias de sobrevivencia y fortalecimiento de las estructuras comunitarias de los grupos indígenas que están orientados hacia la reintegración étnica (Barabas, 2008; Glick, Çaglar, Guldbrandsen, 2006; Levitt, 2001). Las diversas formas de reintegración comunitaria existentes hoy día en el Valle por parte de estos pueblos - como las fiestas, las asambleas regionales y las movilizaciones sociales-que son encabezadas por los grupos indígenas residentes en las colonias, han exaltado la vitalidad de la articulación comunitaria de estos grupos y podemos señalar que ha sido el entorno protestante el que ha funcionado también como aglutinador de ese sentido colectivo. 
Aun con ello, entendemos a estas misiones civilizadoras y al movimiento cristiano internacional como una nueva forma de internacionalización de lo religioso (Levitt, 2001), y como una nueva fase del sistema expansionista estadounidense en su etapa neocolonial. En este sentido, la lógica colonial que ha implantado los valores cristianos en la región, justifica la transición hacia la modernidad de los residentes de ese territorio fronterizo, al instalar un universo moral de logro, del esfuerzo propio y de la búsqueda de superación (Bonino, Álvarez y Craig, 1983), que se vincula abiertamente con la dinámica comercial de la zona y con la industria de agro-exportación que ahí se ha impuesto.

\section{Agradecimientos}

Este documento fue elaborado durante el periodo de estancia posdoctoral realizada en el Instituto de Investigaciones Económicas de la Universidad Nacional Autónoma de México (unam-IIEc). El autor agradece a la Coordinación de Humanidades de la UnAM por el generoso apoyo. También se agradece a los dictaminadores quienes, con sus comentarios, orientaron la ampliación del argumento central que aquí se expone.

\section{Referencias}

Barabas, M. A. (2008). Los migrantes indígenas de Oaxaca en Estados Unidos: fronteras, asociaciones y comunidades. En L. Velasco (Coord.), Migración, fronteras $e$ identidades étnicas transnacionales (pp.171-193). México: El Colegio de la Frontera Norte, Miguel Ángel Porrúa.

Bengoa, J. (2000). La emergencia indígena en América Latina. Chile: Fondo de Cultura Económica.

Bonino, J. M., Álvarez, C. y Craig, R. (1983). Protestantismo y liberalismo en América Lati$n a$. San José, Costa Rica: DeI y Sebila.

Camargo, M. A. (2011). Las formas del movimiento: el papel del vínculo religioso en una región intermedia de migración. En A. Hernández (Coord.), Nuevos caminos de la fe: Prácticas y creencias religiosas en el margen institucional. México: El colegio de la Frontera Norte, El Colegio de Michoacán, Universidad Autónoma de Nuevo León.

Camargo, M. A. (2012). Haciendo vida en esta tierra: El Asentamiento de los triquis en el Valle de San Quintín Baja California. En M. D. París (Coord.), Diáspora Triqui: Violencia política, desplazamiento forzado y migración (pp. 81-92). México: Serie Mundos Rurales, Universidad Autónoma Metropolitana-Unidad Xochimilco, Itaca Editores.

Camargo, M. A. (2015). Migración y cambio religioso. La construcción de "nuevas comunidades" de indigenas migrantes en la frontera noroeste del país (Tesis doctoral). Instituto de Investigaciones Antropológicas, Facultad de Filosofía y Letras, Universidad Nacional Autónoma de México, México. 
Canales, A. (1995). El poblamiento de Baja California 1848-1950. Frontera Norte, 7(13), 5-23.

Demera, J. D. (2008). Trayectorias del protestantismo y redefiniciones étnicas entre los indígenas Guambianos-Colombia. Revista de Ciencias Sociales, 1(20), 107-128.

Gallardo, M. (2010). Reestructuración productiva en la horticultura del Valle de San Quintín, Baja California, y su impacto en la generación del empleo de 1994 a 2008 (Tesis de maestría). El Colegio de la Frontera Norte, México.

Garma, C. (2004). Buscando el espíritu: Pentecostalismo en Iztapalapa y la ciudad de México. México: Universidad Autónoma Metropolitana-Iztapalapa, Plaza y Valdés Editores.

Glick, N., Chaglar, A. y Guldbrandsen, T. C. (2006). Beyond the Ethnic Lens: Locality, Globality, and Born-again Incorporation. American Ethnologist, 33(4), 612-633.

González, L. (2009). Movilidad laboral. Imposición estructural para la incorporación indígena a los mercados de trabajo en contextos globales. Migración y Desarrollo, (13), 47-59.

Guarnizo, L. E. (2010). Notas sobre la movilidad contemporánea del capital y del trabajo. En S. M. Lara (Coord.), Migraciones de trabajo y movilidad territorial (pp. 47-80). México: Conacyt, Miguel Ángel Porrúa.

Heath, H. J. (2011). El malogrado proyecto del ferrocarril peninsular del Distrito Norte de la Baja California, 1887-1892. Estudios Fronterizos, 12(24), 185-221.

Hernández, H. A. (1999). La formación de la frontera norte y el protestantismo. En P. Galeana (Coord.), Nuestra frontera norte (pp.61-79). México: Archivo General de la Nación.

Hernández, H. A. y O’Connor, M. I. (2013). Migración y conversión religiosa entre los mixtecos de Oaxaca. Revista Alteridades, 23(45), 9-23.

Hernández, M. M. (2000). El proceso de convertirse en creyentes: Identidades de familias. Testigos de Jehová en un contexto de migración transnacional. Relaciones, $\operatorname{xхxIx}(83), 17-29$.

Higham, J. (1983). Strangers in the land: Patterns of American Nativism, 1860-1925. Estados Unidos de América: The State University, Rutgers.

Instituto Nacional de Estadística, Geografía e Informática (Inegi). (2010). Censo de población y vivienda. México: Autor.

Jaimes, M. R. (2009). La migración como factor de cambio religioso en Tijuana. En J. C. Ruiz y O. Odgers (Coords.), Migración y creencias: Pensar en las religiones en tiempo de movilidad (pp. 333-360). México: El Colegio de la Frontera Norte, El Colegio de San Luis, Porrúa.

Jordán, F. (1997). El otro México: biografía de Baja California. México: Universidad Autónoma de Baja California.

Lalive, C. (1970). Les protestantismes latino-américans. Un modèle typologique. Archives de Sociologie des Religions, (30), 33-57.

Lara, F. S. M. (2011). Los encadenamientos migratorios en espacios de agricultura intensiva. México: Miguel Ángel Porrúa, El Colegio Mexiquense.

Levitt, P. (2001). Between God, Ethnicity, and Country: An Approach to the Study of Transnational Religion. Cartel presentado en el seminario Transnational Migration: Comparative Perspectives, Princeton University, Estados Unidos de América. 
Marín, R. (1982). La Doctrina Monroe, el Destino Manifiesto y la expansión de Estados Unidos sobre América Latina: El caso de México. Revista Estudios, (4), 117-141.

Marroquín, E. (2007). El conflicto religioso. Oaxaca 1976-1992. México: Universidad Autónoma de México, Centro de Investigaciones Interdisciplinarias en Ciencias y Humanidades, Universidad Autónoma Benito Juárez de Oaxaca, Instituto de Investigaciones Sociológicas.

Martínez, N. C. (2006). Who Defines Indigenous. Identities, Development, Intellectuals and the State in Northern Mexico. Londres, Inglaterra: Rutgers University Press.

Moreno, J. A. (2008). Los valles agrícolas de Baja California: Espacios de agricultura para la exportación. En A. León, B. Canabal y R. Pimienta (Coords.), Migración, poder y procesos rurales (pp. 65-77). México: Universidad Autónoma Metropolitana, Plaza y Valdés.

Ramírez, V. D. (2008). Bajo la sombra del pirul: Memoria histórica del rancho Las Escobas, Valle de San Quintín. México: Instituto de Cultura de Baja California, Editorial Voces de la Península.

Spurr, D. (2014). La retórica del imperio: El discurso colonial en periodismo, escritura de viajes y administración imperial. Santiago de Chile: Ediciones Universidad Alberto Hurtado.

Taylor, H. L. D. (2000). La transformación de Baja California en Estado 1931-1952. Estudios Fronterizos, 1(1), 47-87.

Velasco, L. (2011). Identidad regional y actores: una experiencia de intervención sociológica en el Valle de San Quintín, Baja California. Región y Sociedad, 23(51), 43-70.

Velasco L., Zlolniski, C. y Coubés, M. L. (2014). De jornaleros a colonos: Residencia, trabajo e identidad en el Valle de San Quintín. México: El Colegio de la Frontera Norte.

Zabin, C. (Coord.). (1992). Migración oaxaqueña a los campos agrícolas de California: Un diálogo. Estados Unidos de América: Center for U.S.-Mexican Studies, University of California-San Diego.

Zlolniski, C. 2011. De campamentos a colonias: horticultura de exportación y asentamiento en el Valle de San Quintín, Baja California. Ponencia presentada en el $8^{\circ}$ Congreso Nacional de la Asociación Mexicana de Estudios Rurales, Puebla, Puebla, México.

Abbdel Camargo Martínez

Mexicano. Doctor en Antropología por la Universidad Nacional Autónoma de México (UNAM), maestro en Desarrollo Regional por El Colegio de la Frontera Norte (Colef); cuenta con una especialidad en Políticas Públicas. Su formación como antropólogo la obtuvo en la Escuela Nacional de Antropología e Historia (ENAH). Líneas de investigación: mercados de trabajo, movilidades y estudios sobre frontera. Camargo, A. (2016). De Migrantes a Residentes: Jornaleros agrícolas y su proceso de arraigo en San Quintín. En B. Canabal y M. A. Olivares (Coords.), Sujetos rurales: Retos y nuevas perspectivas de análisis. México: Universidad Autónoma Metropolitana (UAMXochimilco), Itaca. 